\title{
Comparative study of the hemolymph microbiome between live and recently dead American lobsters Homarus americanus
}

\author{
Jibom Jung ${ }^{1}$, Patrick M. Gillevet ${ }^{2}$, Masoumeh Sikaroodi ${ }^{2}$, Jamal Andrews ${ }^{2}$, \\ Bongkeun Song ${ }^{3}$, Jeffrey D. Shields ${ }^{3, *}$
}

\author{
${ }^{1}$ School of Biological Sciences, Seoul National University, Gwanak-gu, Seoul 08826, Republic of Korea \\ ${ }^{2}$ Microbiome Analysis Center, George Mason University, Fairfax, VA 22030, USA \\ ${ }^{3}$ Virginia Institute of Marine Science, College of William \& Mary, Gloucester Point, VA 23062, USA
}

\begin{abstract}
Lobsters and other crustaceans do not have sterile hemolymph. Despite this, little is known about the microbiome in the hemolymph of the lobster Homarus americanus. The purpose of this study was to characterize the hemolymph microbiome in lobsters. The lobsters were part of a larger study on the effect of temperature on epizootic shell disease, and several died during the course of the study, providing an opportunity to examine differences in the microbiomes between live and recently dead (1-24 h) animals. The hemolymph microbiomes of live lobsters was different from those in dead animals and both were different from the tank microbiome in which the animals had been held. The microbiomes of live lobsters were more diverse and had a different suite of bacteria than those from dead animals. The dominant taxa in live lobsters belonged to Flavobacteriaceae and Rhodobacteraceae, whereas Vibrionaceae and Enterobacteriaceae were predominant in the dead lobsters. Although aquarium microbiomes overlapped with the hemolymph microbiomes, there was less overlap and lower abundance of taxa in comparison with hemolymph from live lobsters. Previous studies reporting bacteria in the digestive tract of lobsters suggested that Vibrionaceae and Enterobacteriaceae had invaded the hemolymph via the gut. Our study suggests that hemolymph bacteria abundant in live lobsters do not originate from the tank milieu and comprise a rich, natural, or native background of bacterial constituents.
\end{abstract}

KEY WORDS: Decapoda - Microorganism - Biodiversity - Bacteria - Succession - Vibrio . Gut microbiome $\cdot$ Comparative microbiomes

\section{INTRODUCTION}

The American lobster Homarus americanus is a highly valued commercial species and one of the main fishery products in the world. The ex-vessel landings are around $160000 \mathrm{mt}$ and represent approximately half of world-wide lobster catch (FAO 2018). Because of its abundance, relative ease of capture, maintenance, handling, and large size, the American lobster is also used as a model crus-

${ }^{*}$ Corresponding author: jeff@vims.edu tacean for comparative studies (e.g. Romano et al. 2007, Ma et al. 2008, Waller et al. 2017). However, the microbiomes of the lobster have only recently been studied. The external, or shell microbiomes of the lobster have received the most attention, primarily because of the dysbiosis found in epizootic shell disease (ESD) (Meres et al. 2012, Quinn et al. 2013, Reardon et al. 2018). Bacterial taxa such as Aquimarina, Tenacibaculum, Maribacter, Jannischia, and others are more abundant on diseased

(C) The authors 2021. Open Access under Creative Commons by Attribution Licence. Use, distribution and reproduction are unrestricted. Authors and original publication must be credited. 
animals than on healthy animals. Although there has been no work on the gut microbiome of the American lobster, a recent study has examined how the formation of gut microbiome changes in the European lobster H. gammarus (Holt et al. 2020). In addition, using ultrastructure and specialized staining, Martin et al. (2020) found that American lobsters and several other crustaceans do not have an active microbiome in the midgut region because the food bolus is encased in a peritrophic membrane that excludes bacteria from entering the lumen of the midgut.

As with many marine invertebrates, American lobsters often do not have sterile hemolymph. Previous studies used culture techniques to isolate and identify few culturable bacteria in the lobster hemolymph, including significant pathogens (Cornick \& Stewart 1966, Bartlett et al. 2008), but these methods are highly selective and the results may not be representative of the taxa present in the hemolymph microbiome (Bent \& Forney 2008). Moreover, culture-dependent methods often give negative results, leading to the notion that healthy hemolymph should be sterile. Quinn et al. (2013) examined bacteria in the lobster hemolymph using culture-independent methods (nested-PCR of 16S rRNA and denaturing gradient gel electrophoresis), but reported only a few taxa, and one sample did not have bacteria. In a study using next generation sequencing (NGS) of $16 \mathrm{~S}$ amplicons, the whole hemolymph microbiome of the spiny lobster Panulirus ornatus was found to include a high diversity of potentially symbiotic bacteria (Ooi et al. 2019). Given that spiny lobsters (Infraorder Palinura: Palinuridae) are not closely related to clawed lobsters (Infraorder Astacidea: Nephropidae), and live in tropical and subtropical regions, they are likely to have very different microbiomes. Nonetheless, there have been no NGS studies to date on the microbiome in the hemolymph of $H$. americanus or other nephropid lobsters.

Our objective was to identify the hemolymph microbiome of the American lobster and to characterize its diversity using 16S amplicon NGS. Because the animals were part of a larger, long-term temperature experiment on ESD (see Barris et al. 2018), we had the opportunity to compare the microbiomes of live lobsters and those that had died of natural causes (within $24 \mathrm{~h}$ ) during the experiment. In addition, we analyzed the microbiomes of the aquaria (tank microbiomes) to examine whether the hemolymph microbiomes originated from their external environment.

\section{MATERIALS AND METHODS}

\subsection{Treatment of lobsters}

The capture, care, feeding, and maintenance of Homarus americanus was described in Barris et al. (2018). In that study, we used 65 females and 14 males, 59 with ESD and 20 without shell disease (termed healthy) (total $N=79$ ). The intensity of infection with ESD was categorized visually: 0: healthy, no scarring; light: shell disease $<10 \%$ of body surface with lesions; moderate: shell disease over $11-50 \%$ of the body; and heavy: with shell disease $>50 \%$ of the body. The animals were held individually in $38 \mathrm{l}$ aquaria at either $6^{\circ}(\mathrm{n}=30), 12^{\circ}(\mathrm{n}=25)$, or $18^{\circ} \mathrm{C}(\mathrm{n}=24)$ for $6 \mathrm{mo}$, and other variables such as salinity and water quality were controlled to match normal seawater conditions. Water quality was maintained with $30-50 \%$ water changes weekly. Nine lobsters died during the course of the long-term temperature study; their hemolymph was sampled as described below. In addition, at the end of the experiment, hemolymph samples were taken from 9 haphazardly selected healthy lobsters and swab samples were taken from 39 aquaria in which many of these lobsters had been held.

\subsection{Sampling}

Hemolymph samples were drawn aseptically from the juncture of the basis and the ischium of the $5^{\text {th }}$ walking leg from lobsters at the beginning and end of the experiment and on the day of death for those that died during the experiment. A $95 \%$ ethanol swab was used to sterilize the region prior to bleeding. Several drops of hemolymph from every live animal were plated directly onto marine agar (Difco 2216) and assessed for colony growth after 24 and $48 \mathrm{~h}$. For the microbiome analysis, approximately $100 \mu \mathrm{l}$ hemolymph was taken from each of the 9 haphazardly selected animals, as well as from each of the 9 recently dead lobsters (Table 1), placed in $1.0 \mathrm{ml} 95 \%$ ethanol, placed on ice for a short period, and then frozen at $-80^{\circ} \mathrm{C}$. Because animals were monitored 1-2 times $\mathrm{d}^{-1}$, any dead lobsters had died within 1-24 h of their sampling. Hemolymph samples from live lobsters were taken again after 67 mo in captivity in November 2017. Hemolymph samples of dead lobsters were taken at the time of their death, with most mortalities in July and August 2017. In addition to the hemolymph samples, tank (aquarium) samples were taken from 39 aquaria in which lobsters were held at the end of 
Table 1. Status of hemolymph samples from Homarus americanus used in this study. Dead animals were given a subjective 'smell test' to determine approximately when they died. If the animal smelled rank (dead smell), it was presumed to have died at least $12-24 \mathrm{~h}$ previous to the sample. If the animal smelled like a live animal, it was presumed to have died within the last 1-12 h. ESD: epizootic shell disease; ND: not determined; NA: animal did not die during experiment

\begin{tabular}{|lccclc|}
\hline ID & $\begin{array}{c}\text { Internal } \\
\text { reference } \\
\text { number }\end{array}$ & $\begin{array}{c}\text { Temperature } \\
\text { treatment } \\
\left({ }^{\circ} \text { C) }\right.\end{array}$ & $\begin{array}{c}\text { Disease } \\
\text { condition }\end{array}$ & Status & $\begin{array}{c}\text { Time } \\
\text { since } \\
\text { death }(\mathrm{h})\end{array}$ \\
\hline HM1 & A1 & 6 & ESD & Dead & $12-24$ \\
HM2 & A2 & 6 & ESD & Dead & $<12$ \\
HM3 & A8 & 6 & ESD & Dead & $<12$ \\
HM4 & AA22 & 6 & ESD & Dead & $<12$ \\
HM5 & B10 & 12 & Healthy & Dead & $12-24$ \\
HM6 & B17 & 12 & ESD & Dead & ND \\
HM7 & F1 & 18 & ESD & Dead & $12-24$ \\
HM8 & C8 & 18 & ESD & Dead & ND \\
HM9 & F10 & 18 & ESD & Dead & $<12$ \\
HM10 & AA5 & 6 & Healthy & Alive & NA \\
HM11 & AA15 & 6 & ESD & Alive & NA \\
HM12 & AA16 & 6 & ESD & Alive & NA \\
HM13 & AA17 & 6 & ESD & Alive & NA \\
HM14 & AA18 & 6 & ESD & Alive & NA \\
HM15 & AA19 & 6 & ESD & Alive & NA \\
HM16 & AA21 & 6 & ESD & Alive & NA \\
HM17 & AA23 & 6 & ESD & Alive & NA \\
HM18 & B1 & 12 & ESD & Alive & NA \\
\hline
\end{tabular}

the amplification (Suzuki et al. 1998, Sikaroodi \& Gillevet 2012). Multi-tag pyrosequencing (MTPS) was then used to analyze the samples by creating fusion primers with 16S rRNA primers, a 7 base barcode, and emulsion PCR adaptors as in Sikaroodi \& Gillevet (2012). Each DNA sample was amplified with both-directed and combined 16S rRNA primers; these were subjected to emulsion PCR and sequenced on an Ion Torrent PGM. The BioProject accession number of this study is PRJNA644743.

\subsection{Analyses}

The analyses of the microbiome sequences were conducted following a modified method of Semedo \& Song (2020). Prior to analysis, the amplicon sequence variant (ASV) count data were filtered using the 'FilterAnd Trim' command in DADA2 (Callahan et al. 2016). The 'fastq' files were the experiment in November 2017. These samples consisted of sterile cotton swabs wiped along the inside of each aquarium and placed into sterile microfuge tubes. Hemolymph and tank samples were held on ice for short periods (20-30 min) until frozen at $-80^{\circ} \mathrm{C}$ and shipped to the Microbiome Analysis Center, George Mason University, for further processing. Due to cost constraints, only a subset of hemolymph samples were processed for microbiome analysis, those from the 9 recently dead lobsters and those haphazardly selected from 9 live lobsters at the end of the experiment.

\subsection{Sequencing}

Each sample was extracted using the FastDNA ${ }^{\mathrm{TM}}$ Spin Kit for Soil (MP Biomedicals) to obtain purified DNA samples. The DNA amplification protocol and sequencing methods followed those described by Reardon et al. (2018). Briefly, the primers $27 \mathrm{~F}$ and $355 \mathrm{R}$ were used to amplify the first 2 variable regions of the bacterial 16s ribosomal RNA through PCR. Length heterogeneity (LH)-PCR fingerprinting was used as a quality control to assure linear amplification of the sample using an ABI 3130 XL fluorescent sequencer to check the quality and reproducibility of collated and processed using DADA2 in R v.3.6.1 using a forward read only option. The sequencing results were examined as quality scores versus amplification cycles and were deemed acceptable for further analyses (Fig. S1 in Supplement 1 at www.int-res.com/articles/suppl/d143p147_supp1.pdf). ASVs were identified from each sample, resulting in 327083 sequence reads identified with Silva Reference database v.132 (Quast et al. 2013). Mitochondrial and chloroplast sequences were removed for diversity analysis of microbiomes using Phyloseq (McMurdie \& Holmes 2013). Rarefaction (Fig. S2), abundance-based coverage estimator (ACE), and Shannon indexes were used to estimate $\alpha$-diversity, and principal coordinate analysis ( $\mathrm{PCoA}$ ) with Bray distance was used to examine $\beta$-diversity of the microbiome. 'VennDiagram' (Chen \& Boutros 2011) was used to draw a Venn diagram of the microbiomes, 'ggplot' (Wickham \& Chang 2008) was used to make stacked bar plots of the microbiomes, and 'DESeq2' (Love et al. 2014) was used to compare microbiome from different conditions. PERMANOVA was performed on the Bray-Curtis resemblance matrix derived from Hellinger-transformed ASV counts to test for the effect of lobster status (live vs. dead), temperature treatment, and shell disease condition using the 'adonis2' and 'strata' 
functions (strata=spawn). PERMDISP was conducted to determine whether multivariate dispersion had an effect on the 3 testing conditions. PERMANOVA and PERMDISP analyses were conducted in the 'vegan' R package. The ANOVA feature in 'DESeq2' (Love et al. 2014) was used to compare microbiomes from different conditions and distinguish differences in abundance among bacterial genera between treatment groups. For bar plots, ASVs with a relative abundance $>1 \%$ were used to reduce the complexity of the plot and legend due to rare genera.

\section{RESULTS}

\subsection{General observation}

Culturable bacteria were present in 52.2-86.7\% of the hemolymph samples taken from lobsters (Table 2). There was a significant decrease in the prevalence of bacteremia over time $\left(\chi^{2}=4.680, p=0.031\right)$, but it was only observed in animals held at $6^{\circ} \mathrm{C}$. Colonies growing on marine agar varied in their morphology, but no consistent patterns were observed. Of the hemolymph samples selected for high-throughput sequencing, all but one (HM18) had colony growth consistent with low level bacteremia.

The hemolymph samples from lobsters had diverse members in their microbiomes (Table 3). Collectively, 173 families, 316 genera, and 305 unclassified and 52 classified ASVs of bacteria were present in the hemolymph of live and dead lobsters (Table S1 in Supplement 2 at www.int-res.com/ articles/suppl/d143p147_supp2.xlsx). The diversity of

Table 2. Percentage of lobsters with culturable bacteria present in the hemolymph (bacteremia) at the start and end of a 6 mo temperature experiment. Details of the experimental conditions and temperature treatments are given in Barris et al. (2018)

\begin{tabular}{|c|c|c|}
\hline $\begin{array}{l}\text { Temperature } \\
\text { treatment } \\
\left({ }^{\circ} \mathrm{C}\right)\end{array}$ & $\begin{array}{l}\text { \% Positive at start } \\
\text { (no. positive/ } \\
\text { total no.) }\end{array}$ & $\begin{array}{l}\text { \% Positive at end } \\
\text { (no. positive/ } \\
\text { total no) }\end{array}$ \\
\hline 6 & $86.7(26 / 30)$ & $60.9(14 / 23)^{\mathrm{a}}$ \\
\hline 12 & $52.2(12 / 23)$ & $50.0(9 / 18)$ \\
\hline 18 & $83.3(20 / 24)$ & $68.8(11 / 16)^{\mathrm{b}}$ \\
\hline \multicolumn{3}{|c|}{$\begin{array}{l}\text { a Significantly lower prevalence of bacteremia than at } \\
\text { start }\left(\chi^{2}=4.680, p=0.031\right) \\
\text { bThe lower sample size in this treatment reflects a con- } \\
\text { taminant present in the initial media. When possible, } \\
\text { the animals were resampled using new media }\end{array}$} \\
\hline
\end{tabular}

the microbiome was somewhat higher in the hemolymph of live than in dead lobsters (Fig. S2, Table 3). In addition, only 3 of 9 hemolymph samples from dead animals had diversity indices similar to those found in live lobsters. The lowest richness ( $\mathrm{ACE}=5$ and 9) was observed in the hemolymph of 2 dead lobsters (HM2 and HM8), whereas the highest richness (ACE = 193) was from the hemolymph of a live lobster (HM17; Tables 2 \& S1). Although some hemolymph samples from dead lobsters had a relatively high diversity, as indicated by their high ACE index (e.g. HM5, HM6, and HM9), they had relatively low Shannon diversity values and differed from those values in the live hemolymph samples. This tendency in the hemolymph samples from dead lobsters indicates that they had lower evenness despite higher richness. These differences were notable for the proportionally higher abundance of Vibrio (HM5, HM9) and other bacterial species (HM5, HM6, HM9) which were not common in hemolymph samples from live lobsters. In addition, PERMANOVA showed significant differences among microbiomes from live versus dead lobsters $\left(F_{1,39}=2.912, \mathrm{p}<0.001\right)$ and different temperatures $\left(F_{1,39}=1.338, \mathrm{p}<0.05\right)$ with homogenous dispersions. However, the sample sizes for animals at 12 and $18^{\circ} \mathrm{C}$ were too low to draw inferences. The presence of ESD did not have significant effect on microbiome compositions $\left(F_{1,39}=1.084, \mathrm{p}>0.05\right)$.

The microbiomes present in the hemolymph of live and dead lobsters exhibited communities different from those in the 39 tank (aquarium) microbiomes (Fig. 1). There were 186 families, 316 genera, and 339 ASVs present in the tank microbiomes (Table S2), and 43 families, 64 genera, and 68 ASVs overlapped with the hemolymph microbiomes (50 overlapped with hemolymph from live animals and 48 overlapped with hemolymph from dead animals) (Fig. 2).

Although tank microbiomes formed different groups depending on temperature (Fig. 1), there were too few hemolymph samples processed from animals held at different temperatures to make inferences about temperature. Nonetheless, there was no overlap in the PCoA values for microbiomes from both live and dead lobsters compared with those in tank microbiomes.

Several bacterial taxa in tank water overlapped with the taxa in lobster hemolymph (Fig. 2, Table S3). Among the 30 taxa shared between tank microbiomes and hemolymph microbiomes of live and dead lobsters, Methylobacterium adhaesivum and Halioglobus sp. were slightly more abundant in live lobsters, Aquimarina sp. was more abundant in dead 
Table 3. Condition, number of sequences, observed species richness, abundance-based coverage estimator (ACE, mean $\pm \mathrm{SD}$ ) and Shannon statistics for the microbiomes from individual lobsters

\begin{tabular}{|c|c|c|c|c|c|}
\hline Condition & Sample & $\begin{array}{c}\text { No. of } \\
\text { sequences }\end{array}$ & $\begin{array}{l}\text { Observed } \\
\text { richness }\end{array}$ & $\mathrm{ACE}$ & Shannon \\
\hline \multirow[t]{9}{*}{ Dead } & HM1 & 3409 & 14.7 & $21 \pm 2$ & 1.03 \\
\hline & HM2 & 7870 & 3.4 & $5 \pm 1$ & 0.83 \\
\hline & HM3 & 7570 & 16.3 & $17 \pm 1$ & 2.40 \\
\hline & HM4 & 4815 & 19.5 & $24 \pm 2$ & 1.59 \\
\hline & HM5 & 4555 & 71.2 & $85 \pm 5$ & 3.20 \\
\hline & HM6 & 8446 & 107.4 & $160 \pm 6$ & 2.66 \\
\hline & HM7 & 6132 & 24.6 & $30 \pm 3$ & 1.88 \\
\hline & HM8 & 6242 & 8.6 & $9 \pm 1$ & 1.82 \\
\hline & HM9 & 6334 & 112.7 & $154 \pm 6$ & 3.72 \\
\hline \multirow[t]{9}{*}{ Live } & HM10 & 1416 & 112.7 & $120 \pm 5$ & 4.31 \\
\hline & HM11 & 1740 & 75.0 & $80 \pm 4$ & 3.60 \\
\hline & HM12 & 1175 & 76.5 & $80 \pm 4$ & 3.75 \\
\hline & HM13 & 1140 & 101.0 & $104 \pm 5$ & 4.33 \\
\hline & HM14 & 1771 & 101.8 & $106 \pm 5$ & 4.28 \\
\hline & HM15 & 801 & 66.3 & $67 \pm 4$ & 3.87 \\
\hline & HM16 & 1710 & 73.9 & $79 \pm 4$ & 2.99 \\
\hline & HM17 & 3395 & 154.9 & $188 \pm 7$ & 4.57 \\
\hline & HM18 & 2575 & 77.5 & $89 \pm 5$ & 3.01 \\
\hline \multirow[t]{39}{*}{ Tank } & TA1 & 4972 & 65.3 & $76 \pm 4$ & 3.36 \\
\hline & TA2 & 3769 & 56.7 & $64 \pm 4$ & 3.21 \\
\hline & TA3 & 11057 & 106.2 & $154 \pm 6$ & 3.69 \\
\hline & TA4 & 11175 & 91.9 & $133 \pm 5$ & 3.62 \\
\hline & TA5 & 8336 & 96.1 & $124 \pm 5$ & 3.76 \\
\hline & TA6 & 6749 & 97.4 & $123 \pm 5$ & 3.92 \\
\hline & TA7 & 9134 & 109.8 & $152 \pm 6$ & 4.09 \\
\hline & TA8 & 6612 & 87.2 & $108 \pm 5$ & 3.56 \\
\hline & TA9 & 10275 & 105.6 & $152 \pm 6$ & 3.68 \\
\hline & TA10 & 5934 & 79.1 & $102 \pm 5$ & 3.58 \\
\hline & TA11 & 10059 & 112.8 & $158 \pm 6$ & 3.96 \\
\hline & TA12 & 9019 & 90.8 & $130 \pm 6$ & 3.42 \\
\hline & TA13 & 5209 & 103.4 & $128 \pm 6$ & 3.99 \\
\hline & TA14 & 5216 & 93.0 & $118 \pm 5$ & 3.89 \\
\hline & TA15 & 5249 & 105.6 & $138 \pm 6$ & 3.99 \\
\hline & TA16 & 5431 & 94.1 & $123 \pm 6$ & 3.70 \\
\hline & TA17 & 5462 & 109.8 & $144 \pm 6$ & 4.00 \\
\hline & TA18 & 4816 & 117.4 & $141 \pm 6$ & 4.22 \\
\hline & TA19 & 6522 & 116.7 & $158 \pm 6$ & 3.93 \\
\hline & TA20 & 3089 & 98.0 & $115 \pm 5$ & 4.02 \\
\hline & TA21 & 5221 & 109.7 & $145 \pm 6$ & 3.97 \\
\hline & TA22 & 4692 & 72.8 & $91 \pm 5$ & 3.24 \\
\hline & TA23 & 3205 & 84.8 & $99 \pm 5$ & 3.57 \\
\hline & TA24 & 4707 & 91.8 & $112 \pm 5$ & 4.10 \\
\hline & TA25 & 4944 & 123.6 & $149 \pm 6$ & 3.97 \\
\hline & TA26 & 5211 & 75.7 & $98 \pm 5$ & 3.23 \\
\hline & TA27 & 2872 & 75.7 & $83 \pm 4$ & 3.73 \\
\hline & TA28 & 4409 & 65.3 & $78 \pm 4$ & 3.22 \\
\hline & TA29 & 3648 & 82.7 & $98 \pm 5$ & 3.52 \\
\hline & TA30 & 2893 & 109.2 & $125 \pm 6$ & 4.20 \\
\hline & TA31 & 4527 & 75.5 & $95 \pm 5$ & 2.99 \\
\hline & TA32 & 3489 & 114.4 & $132 \pm 6$ & 4.15 \\
\hline & TA33 & 2833 & 65.7 & $76 \pm 4$ & 2.96 \\
\hline & TA34 & 3964 & 105.2 & $123 \pm 5$ & 4.03 \\
\hline & TA35 & 3787 & 75.6 & $88 \pm 5$ & 3.50 \\
\hline & TA36 & 2877 & 91.6 & $103 \pm 5$ & 3.85 \\
\hline & TA37 & 3629 & 87.9 & $100 \pm 5$ & 3.75 \\
\hline & TA38 & 4525 & 111.1 & $136 \pm 6$ & 4.00 \\
\hline & TA39 & 5043 & 98.2 & $128 \pm 6$ & 3.28 \\
\hline
\end{tabular}

lobsters, and Anderseniella sp., Marinicella sp., Paraglaciecola sp., and Chitinophagales sp. were more abundant in tank samples. Among the 20 taxa shared between the tank microbiomes and those in the hemolymph of live lobsters, Myxococcales sp. was slightly more abundant in live lobsters. Among the 18 taxa shared between tank samples and hemolymph from dead lobsters, Vibrio sp., Citrobacter sp., Photobacterium profundum, and $P$. indicum were more abundant in dead lobsters.

\subsection{Patterns of the hemolymph microbiome}

In total, the hemolymph microbiome of live lobsters consisted of 242 distinct ASVs. The 3 most diverse families were Flavobacteriaceae, Rhodobacteraceae, and Burkholderiaceae, with many ASVs represented by unclassified genera and species. The 3 most diverse genera were Bacteroides, Corynebacterium, and Methylobacterium (Table S1). The 5 most abundant bacterial families in the hemolymph of live lobsters were Flavobacteriaceae, Rhodobacteraceae, Beijerinckiaceae, Saprospiraceae, and Pseudomonadaceae (Fig. 3A). The abundance of genera was relatively evenly distributed with taxa in the Flavobacteraceae, Alkalimarinus, Ulvibacter, Methylbacterium, Gammaproteobacteraceae, and Loktenella, as well as Halloglobus, Arenicella, and Sapropiraceae (Figs. 3B \& 4).

In total, the microbiome in the hemolymph of dead lobsters consisted of 232 ASVs, i.e. a similar diversity to those in the live animals, but as noted above, the relative abundances of several taxa were very high. The 3 most abundant families were Flavobacteriaceae, Rhodobacteraceae, and Vibrionaceae, as well as many ASVs representing unclassified genera and species. The 2 most diverse genera were Vibrio and Colwellia (Table S1). Six families-Vibrionaceae, Entero- 


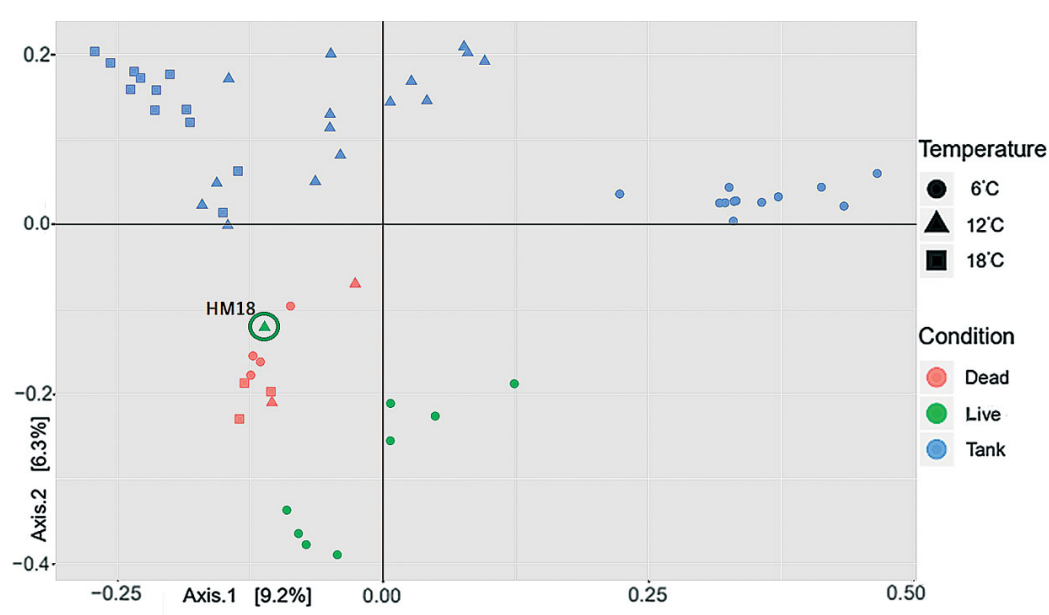

Fig. 1. Principal coordinate analysis of the microbiome of the hemolymph of Homarus americanus and tank, by condition and temperature. Differences between HM18 and the microbiome of dead animals are noted in Section 4
Oceanospirillum, Colwellia, and Arcobacter (Figs. 3B \& 4). These genera were not present or were present at very low levels in the hemolymph of live animals. The microbiomes of 2 dead lobsters (HM4 and HM6) were especially dominated by Citrobacter, Oceanospirillum, and Candidatus Hepatoplasma.

The hemolymph microbiome of dead and live lobsters were significantly different at the genus level (Fig. 5; DESeq ANOVA). Many genera, such as Flavobacteriaceae, Maribacter, Arenicella, Granulosiccus, and Methylobacterium, were much more abundant in the hemolymph of live animals than in that from dead animals, whereas the dominant genera in dead lobster such as Vibrio, Photobacterium, Oceanospirillum, Citrobacter, and Hepatoplasma were significantly more abundant compared to hemolymph of live lobsters.

\section{DISCUSSION}

Using next generation sequencing of $16 \mathrm{~S}$ amplicons, we confirmed the presence of a diverse microbiome in all 18 Homarus americanus hemolymph samples. Live lobsters typically have culturable bacteria in their hemolymph (bacteremia) in a large proportion of their populations (Dove et al. 2005, Bartlett et al. 2008, Shields et al. 2012, Quinn et al. 2013), but here we show a much higher diversity than previously known. We have shown that the hemolymph of lobsters can have a richly diverse microbiome, with as many as 52 families present in one animal (Table S1). Moreover, the hemolymph microbiome appears to be quite different from that on the shell (cf. Meres et al. 2012) and in the milieu of the aquarium (this study). The potential effects of these hemolymph 'infections' remain largely unknown; they could be commensals, mutualists, sublethal parasites, or pathogens (such as Aerococcus viridans var. homari, causative agent of gaffkemia). The diversity of this community should be further characterized to improve our understanding of how they contribute to lobster health and disease resistance.

Early research on the bacterial flora of the shell and hemolymph of decapod crustaceans, including lobsters, postulated that the source of hemolymph infections was either via the 'contaminated' shell or through the gut (for review, see Shields et al. 2006, Citrobacter, Candidatus Hepatoplasma, Aquimarina, 
$\varangle$
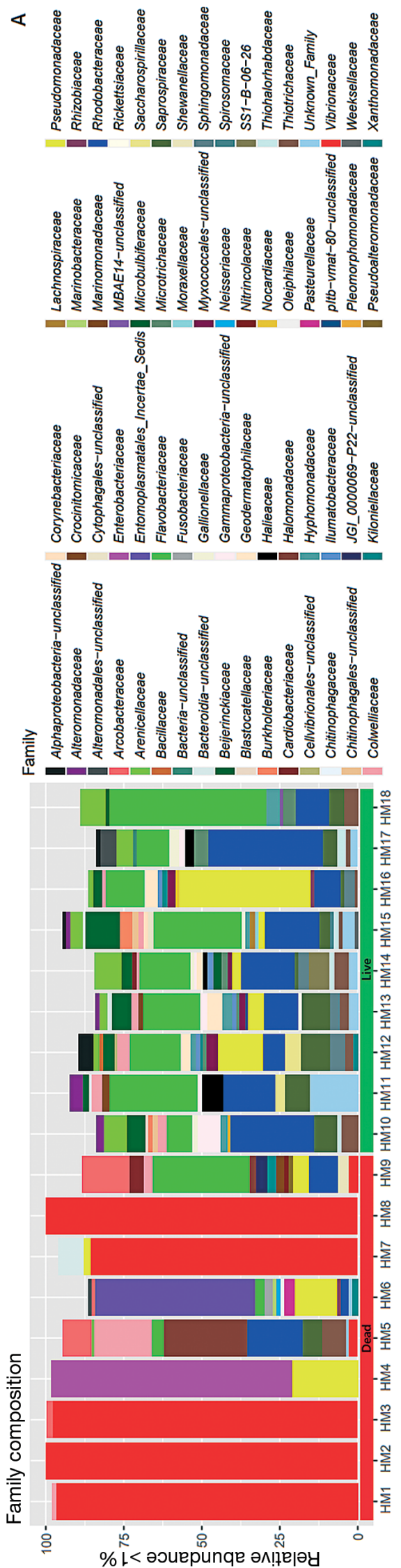

$\infty$

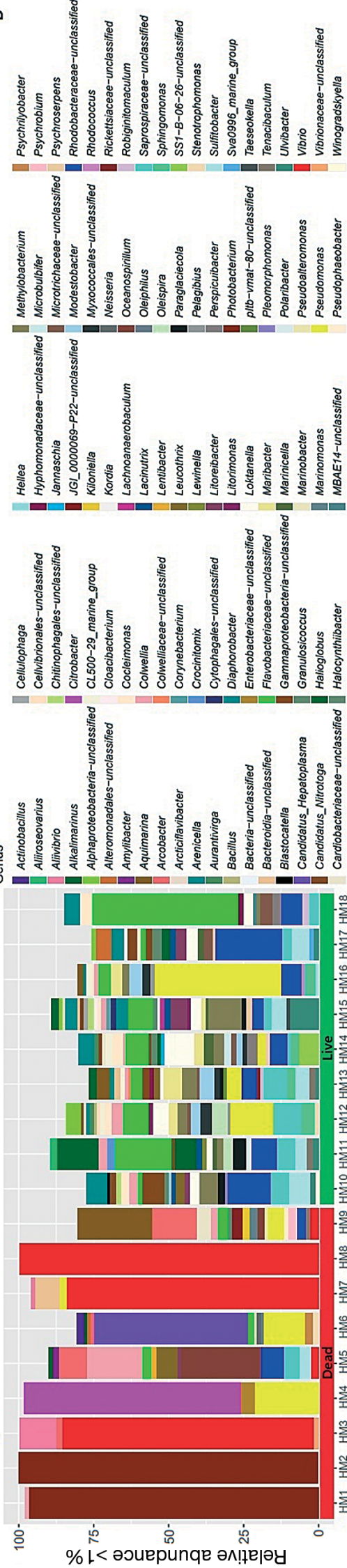

u<smiles>[CH]C#C[Si](C)(C)[Si]#C</smiles>

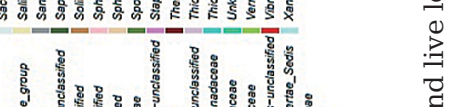

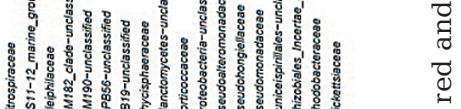

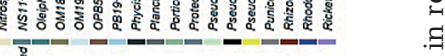

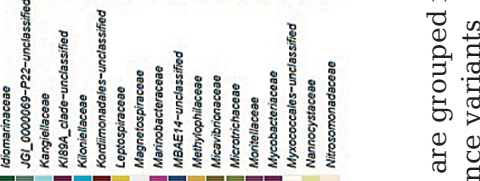

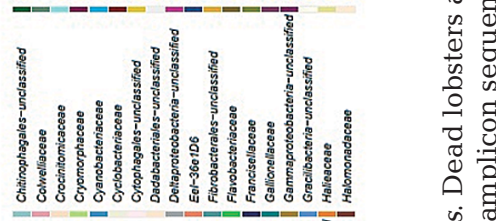

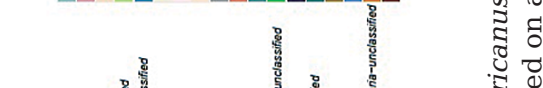

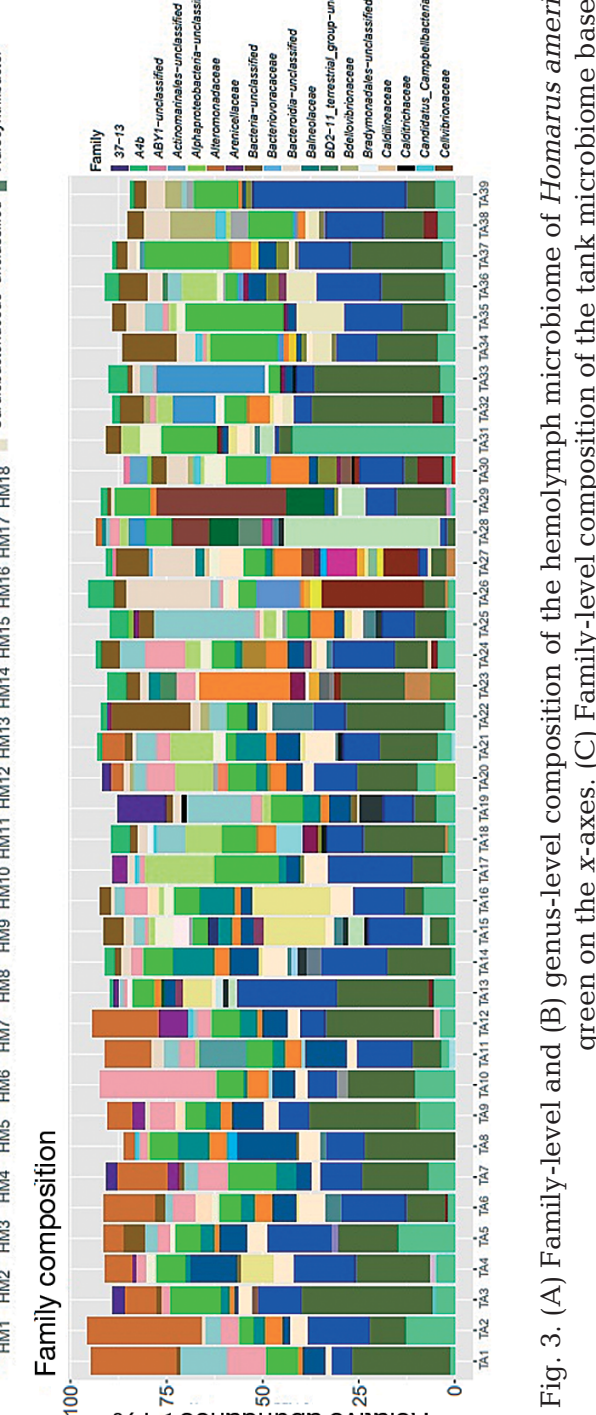




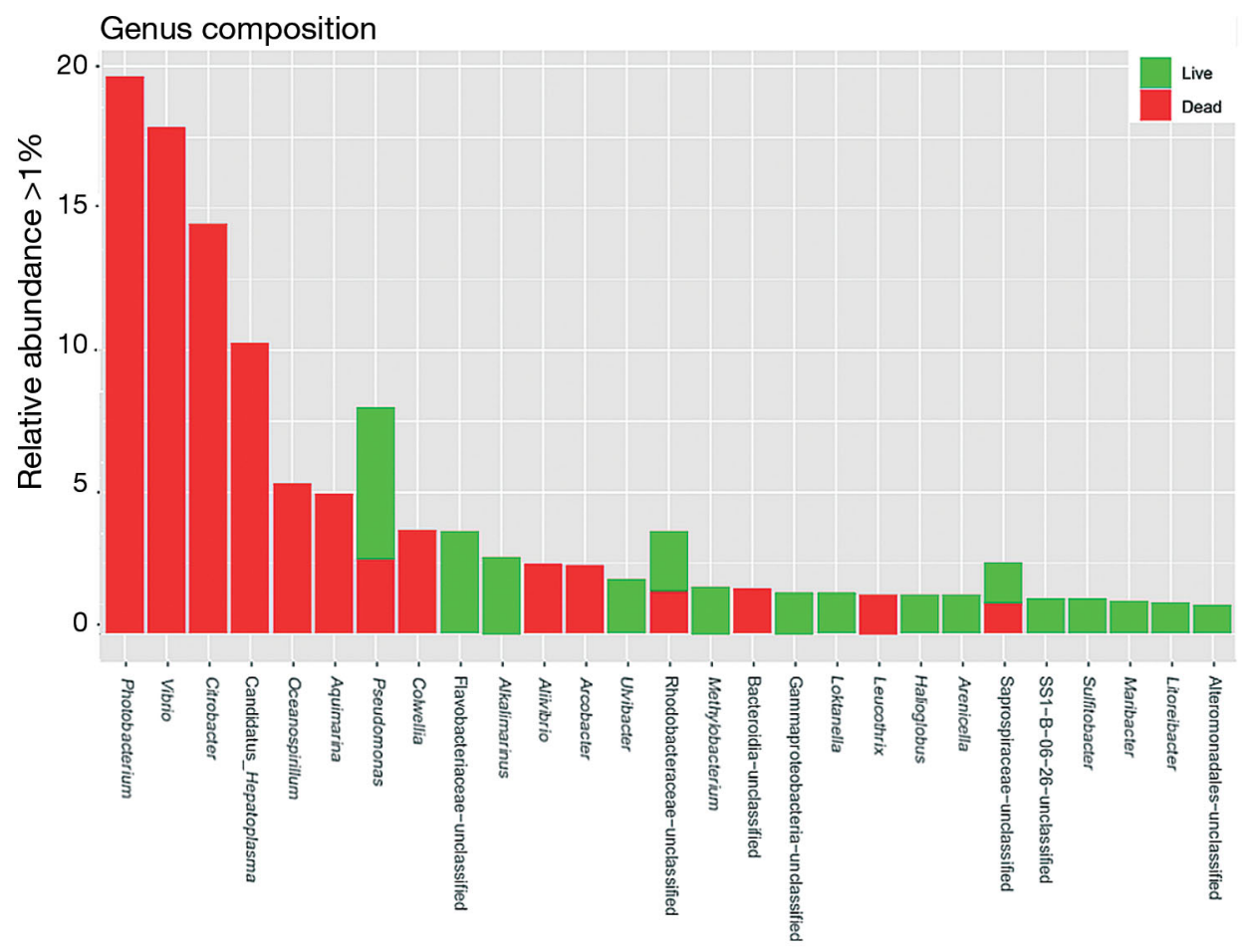

Fig. 4. Genus-level composition in hemolymph microbiome in relation to host status (dead or alive) of the lobster Homarus americanus

Significant changes
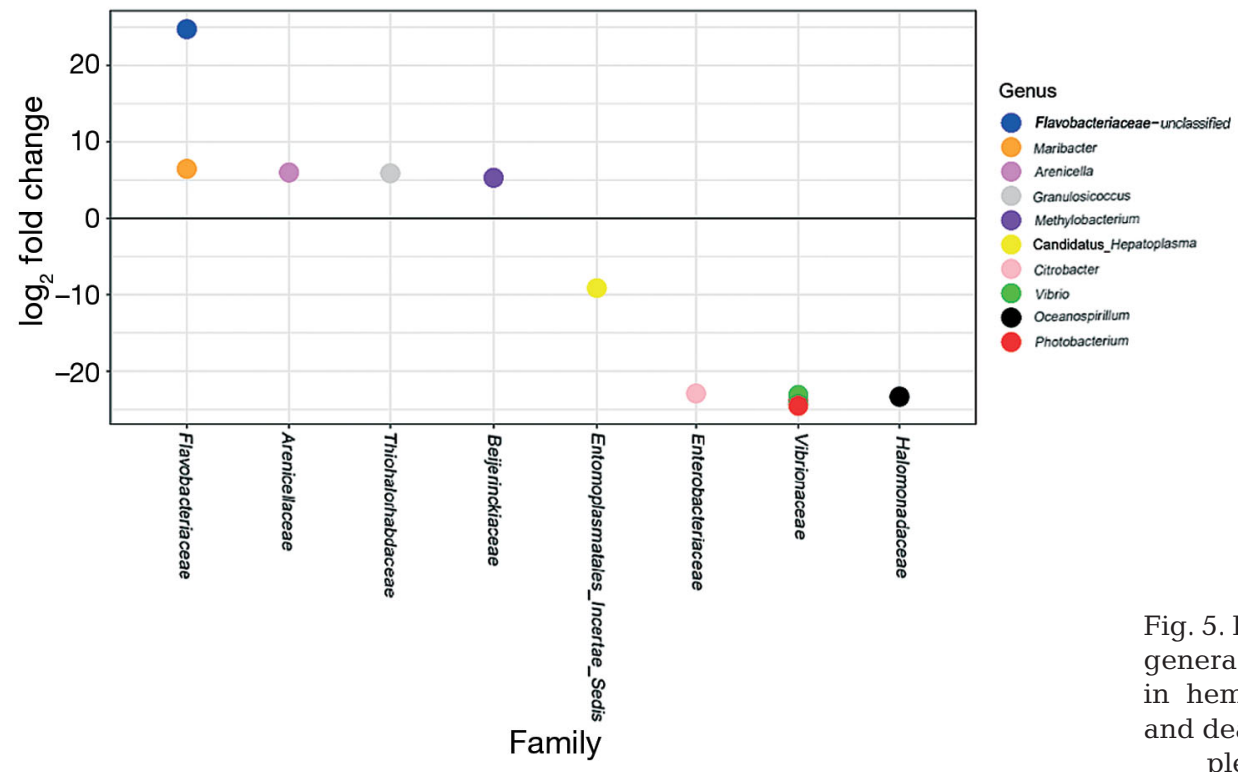

Fig. 5. DESeq ANOVA analysis showing genera that were significantly enriched in hemolymph microbiomes from live and dead lobsters. Above line: live samples, below line: dead samples

Shields \& Overstreet 2007). Our data support that the microbiome in the hemolymph is distinctly different from that on the cuticle (cf. Meres et al. 2012). This is not surprising considering that the shell is exposed to the natural environment and in an external milieu different from that of the hemolymph, which is itself an intimate tissue bathing the internal organs of the host lobster. More importantly, our data indicate that the microbiome in lobster hemolymph rapidly changes when the host lobster dies.

We conjecture that changes in the microbiome in the hemolymph of dead animals arise from invasion through the lobster gut. The gut microbiome of the American lobster $H$. americanus has received little attention. Nonetheless, in a recent study, juvenile sea-reared European lobsters $H$. gammarus had a 
very different gut microbiome than those reared in aquaria (Holt et al. 2020). Early benthic stages reared at sea had more Vibrio spp. and Photobacterium spp. than those reared on land. However, the microbiome present on the feed given to lobsters was not assessed. This is important because Martin et al. (2020) showed that the lumen of the midgut of American lobsters, as well as 5 other crustaceans from disparate taxa, have no microbiome per se. The microbiome is solely present within the food bolus which is entirely encased by the peritrophic membrane. Indeed, the lack of bacteria in the lumen of the midgut has been well documented in other crustaceans (Boyle \& Mitchell 1978, Sleeter et al. 1978, Johnson 1980). Collectively, these findings have implications for lobster health. For example, the hemolymph of dead lobsters appears more like that of the food bolus in that the pathogens are either entering through the food or arise from the food as a consequence of mortality. Another implication is that the gut microbiome may be comprised entirely of what is eaten by the lobsters and not by anaerobic fermenters that are normally present in vertebrate digestive systems and whose members are probiotic species providing essential nutrients.

In terrestrial studies of carcasses, changes in the microbial community are known to follow a rapid sequence of succession, loss of diversity, and increase in abundance of certain taxa within the Proteobacteria and Firmicutes (Pechal et al. 2013). In our study, the hemolymph microbiome in dead lobsters also showed a decline in diversity and shift in specific taxa (Vibrionaceae and Enterobacteriaceae) compared to the hemolymph of live lobsters. This change also appeared to follow a process of succession in relation to post-mortem change and decomposition. The succession appears to occur very rapidly and includes overgrowth by a few dominant taxa.

The hemolymph microbiome of live lobsters is different from that of the tank microbiome and is suggestive of that fact the lobsters have a native or natural microbiome that does not originate directly from the environment. Although there was some overlap in the taxa present in the microbiomes among groups (live, dead, tank), differences in the abundance levels and composition were evident. For example, the most abundant taxa present in the hemolymph of live lobsters such as Flavobacteriaceae and Pseudomonas were not present or were present at very low levels in tank microbiomes.

The correlation between hemolymph microbiomes in dead lobsters and tank microbiomes was less clear. Several abundant ASVs in the hemolymph of dead lobsters were present in the tank samples; however, differences between these groups may have been due to the ability of different taxa to invade and grow as saprobes in the tissues of dead lobsters. Moreover, we did not find a shift in the dominant bacterial community in dead lobsters to be more like that in tank samples. There was, however, a notable flaw in our study as we did not match samples from dead lobsters taken at the same time as tank samples. Nonetheless, the dominant taxa in the tank samples were not the dominant taxa in hemolymph samples from live or dead lobsters.

The hemolymph microbiome of one live lobster (HM18) appeared similar to that of a dead lobster (Fig. 1). The family Flavobacteriaceae and its unclassified genera accounted for about half of the relative abundance of bacteria present in the hemolymph of this animal (Fig. 2B). This pattern was observed in the hemolymph from several dead lobsters, where few taxa were dominant. However, with one exception (HM9), the dominant taxa in dead lobster samples was not Flavobacteriaceae. Notably, HM18 was held at $12^{\circ} \mathrm{C}$, whereas the other animals were held at $6^{\circ} \mathrm{C}$. Although temperature is important in lobster homeostasis, it may not be modulating diversity in the hemolymph of lobsters. In the tropical ornate spiny lobster Panulirus ornatus, a $6 \mathrm{~d}$ increase to $34^{\circ} \mathrm{C}$ $\left(+6^{\circ} \mathrm{C}\right.$ above ambient) had no effect on hemolymph microbiomes (OTUs, richness, diversity indices) compared to those from animals held at ambient temperature $\left(28^{\circ} \mathrm{C}\right)$, even the though those held at $34^{\circ} \mathrm{C}$ began to exhibit mortality after $4 \mathrm{~d}$ (Ooi et al. 2019). Given that lobsters and other crustaceans are often held at somewhat elevated temperatures for commercial shipment, additional studies are needed to determine how temperature affects hemolymph microbiome of these crustaceans.

In most cases, the hemolymph of dead lobsters had a lower microbial richness with the overgrowth or dominance of a few species of Vibrionaceae. Vibrios are common constituents of the marine environment and many are reported as pathogens of aquatic organisms, including lobsters (Tall et al. 2003). However, given the surprisingly low abundance and diversity of vibrios observed in the hemolymph of live lobsters, the role of these bacteria as pathogens or saprobes should be better established. The patterns in diversity in the hemolymph of dead lobsters are likely the result of sublethal stress leading to mortality with the subsequent decomposition of hemolymph through bacterial degradation.

Five families were highly represented in the hemolymph of the live lobsters. The presence of Flavobac- 
teriaceae and Rhodobacteraceae in live hemolymph samples but not in dead samples suggests a possible commensal relationship between these families and the lobster host. Members of Flavobacteriaceae have been reported as insect symbionts (Bernardet \& Nakagawa 2006). One genus of Flavobacteriaceae, Aquimarina, is regarded as a causal agent of ESD in the American lobster (Chistoserdov et al. 2012, Quinn et al. 2012, 2013). Although highly prevalent on the shell of diseased lobsters (data not shown), it was only found in one hemolymph sample (HM9) from a live lobster. However, several other members of the Flavobacteriaceae were present, although their roles in the hemolymph or lobster health remain to be determined. Members of the Rhodobacteraceae are aquatic photo- and chemoheterotrophs as well as symbionts in other organisms (Pujalte et al. 2014). Rhodobacteraceae has been reported as changing in richness in marine organisms in relation to temperature (Stratil et al. 2013, Ooi et al. 2019), but the role of this taxon in the lobster hemolymph is unknown. Methylobacterium spp. are members of the Beijerinckiaceae that are facultative methyltrophs (Lidstrom \& Chistoserdova 2002, Tamas et al. 2014); the genus was abundant in the hemolymph of live lobsters and may use methylated compounds derived from algae and seaweed, but their role in lobster health is unknown. Pseudomonadaceae is abundant in both live and dead lobsters. Members of this taxon have been reported in diseased and dead insects (Jurat-Fuentes \& Jackson 2012), and this may be because they are rapid colonizers with the ability to use diverse energy sources (Palleroni 1981, 2008).

Six families were very abundant in the hemolymph of dead lobsters. Vibrionaceae are facultative anaerobes and infamous as pathogens of many marine organisms (Farmer 2006, Wendling et al. 2014, Sun et al. 2017, Williams et al. 2017). In the present study, several genera in the family (Vibrio, Photobacterium, and Aliivibrio) were present in the hemolymph of dead animals, but not in the hemolymph from live animals. As indicated previously, it is likely that they invaded the dead or dying animal through invasion of the gut. Enterobacteriaceae are also facultative anaerobes and pathogens (Donnenberg 2015). In the present study, Citrobacter was found in the hemolymph of dead lobsters, although their role in lobster health or disease resistance is unclear. Arcobacter spp., a genus in Arcobacteraceae, have been reported as pathogens of fish and mollusks (Fouz et al. 2000, Zhao et al. 2009, Pérez-Pascual et al. 2017, Burioli et al. 2018). They have been reported in biofilms associated with lobsters (Welsh et al. 2011) and as in- dicators of fecal pollution (Collado et al. 2008); hence, they may be opportunistic invaders in this system.

Two studies have examined the bacterial community present in the hemolymph of the American lobster. In a study focused on cultivable bacteria, Bartlett et al. (2008) found several species that were absent in the present study, including Staphylococcus epidermis, S. equorum, S. warnerii, S. xylosus, Delftia (Pseudomonas) acidovorans, Enterococcus faecalis, Hafnia alvei, Klebsiella pneumonia, Pseudomonas putida, and Vibrio fluvialis. In a study using high-throughput sequencing, Quinn et al. (2013) also found several species that were absent in our study, including Weissella paramesenteroides, D. acidovorans, Pelomonas aquatica, Geobacillus tepidamans, Marinosulfonomonas methylotropha, and Brochothrix thermosphacta. At the genus level, Weissella, Novosphingobium, Ralstonia, Pelomonas, Alphaproteobacterium, Hyphomicrobium, Sediminibacterium, Deltaproteobacterium, Geobacillus, Marinosulfonomonas, and Brochothrix were not found in our samples. In both studies, the lobsters were from nearby locations around Rhode Island (Bartlett et al. 2008) and eastern Long Island Sound (Quinn et al. 2013); thus, spatial differences probably do not contribute to these differences. Other factors such as water temperature, time in captivity, handling stress, etc., likely contributed to the differences observed among these studies.

\section{CONCLUSIONS}

We found a high diversity in the hemolymph microbiomes of live American lobsters. The diversity and composition of microbiomes changed when lobsters died, apparently shifting to a more saprobic bacterial community comprised of potential facultative pathogens. The hemolymph microbiome from live lobsters was different from the tank microbiomes and consisted of several families with known symbiotic relationships in other host taxa. Hemolymph from dead lobsters had lower diversity than that from live animals and was mostly dominated by several bacteria rarely found in live lobsters. Tank microbiomes and hemolymph microbiomes of live and dead lobsters overlapped slightly. However, the most abundant ASVs present in the hemolymph of live lobsters were not found in tank samples, and this result suggests that these abundant ASVs did not originate from the external environment. In addition, the ASVs in the hemolymph of dead lobsters were found in tank samples, albeit at low abundance. We speculate that the 
bacteria in the hemolymph of dead lobsters originates from the food in the gut, as there were similarities between the microbiome of the gut of European lobsters with that observed in the hemolymph of dead animals. To understand how the hemolymph microbiome is assembled, further research is needed into how lobsters control the hemolymph microbiome, how the succession of hemolymph microbiome proceeds when a lobster dies, and what role these microorganisms play in the hemolymph.

Acknowledgements. We thank Dr. T. Pugh and M. Trainor at the Massachusetts Division of Marine Fisheries for providing the lobsters used in this study. We also thank W. Kim at Seoul National University for providing visiting support to J.J. to conduct this study. The authors thank S. G. Fortin for help with the analysis of the microbiome. Part of this work was funded by the Saltonstall-Kennedy Program, Award NA14NMF4270044 to J.D.S. This is contribution \#3969 from the Virginia Institute of Marine Science.

\section{LITERATURE CITED}

Barris BN, Shields JD, Small HJ, Huchin-Mian JP and others (2018) Laboratory studies on the effect of temperature on epizootic shell disease in the American lobster, Homarus americanus. Bull Mar Sci 94:887-902

Bartlett SL, Wooster GA, Sokolowski MS, Dove ADM, Bowser PR (2008) Naturally occurring bacteraemia in American lobsters, Homarus americanus Milne-Edwards, in Long Island Sound. J Fish Dis 31:19-25

Bent SJ, Forney LJ (2008) The tragedy of the uncommon: understanding limitations in the analysis of microbial diversity. ISME J 2:689-695

Bernardet JF, Nakagawa Y (2006) An introduction to the Family Flavobacteriaceae. In: Dworkin M, Falkow S, Rosenberg E, Schleifer KH, Stackebrandt E (eds) The prokaryotes: a handbook on the biology of bacteria. Springer, New York, NY, p 455-480.

Boyle PJ, Mitchell R (1978) Absence of microorganisms in crustacean digestive tracts. Science 200:1157-1159

* Burioli EAV, Varello K, Trancart S, Bozzetta E, Gorla A, Prearo M, Houssin M (2018) First description of a mortality event in adult Pacific oysters in Italy associated with infection by a Tenacibaculum soleae strain. J Fish Dis 41: 215-221

*allahan BJ, McMurdie PJ, Rosen MJ, Han AW, Johnson AJA, Holmes SP (2016) DADA2: high-resolution sample inference from Illumina amplicon data. Nat Methods 13: 581-583

Chen H, Boutros PC (2011) VennDiagram: a package for the generation of highly-customizable Venn and Euler diagrams in R. BMC Bioinformatics 12:35

Chistoserdov AY, Quinn RA, Gubbala SL, Smolowitz R (2012) Bacterial communities associated with lesions of shell disease in the American lobster, Homarus americanus Milne-Edwards. J Shellfish Res 31:449-462

Collado L, Inza I, Guarro J, Figueras MJ (2008) Presence of Arcobacter spp. in environmental waters correlates with high levels of fecal pollution. Environ Microbiol 10: 1635-1640

Cornick JW, Stewart JE (1966) Microorganisms isolated from the hemolymph of the lobster (Homarus americanus). J Fish Res Board Can 23:1451-1454

* Donnenberg MS (2015) Enterobacteriaceae. In: Bennett JE, Dolin R, Blaser MJ (eds) Mandell, Douglas, and Bennett's principles and practice of infectious diseases. Saunders, Collingwood, p 2503-2517.

* Dove ADM, Allam B, Powers JJ, Sokolowski MS (2005) A prolonged thermal stress experiment on the American lobster, Homarus americanus. J Shellfish Res 24:761-765

*FAO (2018) The state of world fisheries and aquaculture 2018. FAO, Rome. www.fao.org/3/i9540en/i9540en.pdf

* Farmer JJ (2006) The Family Vibrionaceae. In: Dworkin M, Falkow S, Rosenberg E, Schleifer KH, Stackebrandt E (eds) The prokaryotes. Springer, New York, NY, p 495-507.

Fouz B, Toranzo AE, Milan M, Amaro C (2000) Evidence that water transmits the disease caused by the fish pathogen Photobacterium damselae subsp. damselae. J Appl Microbiol 88:531-535

* Holt CC, van der Giezen M, Daniels CL, Stentiford GD, Bass D (2020) Spatial and temporal axes impact ecology of the gut microbiome in juvenile European lobster (Homarus gammarus). ISME J 14:531-543

Johnson PT (1980) Histology of the blue crab, Callinectes sapidus: a model for the Decapoda. Praeger, New York, NY

Jurat-Fuentes JL, Jackson TA (2012) Bacterial entomopathogens. In: Fernando EV, Harry KK (eds) Insect pathology. Academic Press, Cambridge, p 265-349.

Lidstrom ME, Chistoserdova L (2002) Plants in the pink: cytokinin production by Methylobacterium. J Bacteriol 184:1818

\% Love MI, Huber W, Anders S (2014) Moderated estimation of fold change and dispersion for RNA-seq data with DESeq2. Genome Biol 15:550

*Ma M, Chen R, Sousa GL, Bors EK and others (2008) Mass spectral characterization of peptide transmitters/hormones in the nervous system and neuroendocrine organs of the American lobster Homarus americanus. Gen Comp Endocrinol 156:395-409

* Martin GG, Natha Z, Henderson N, Bang S, Hendry H, Loera Y (2020) Absence of a microbiome in the midgut trunk of six representative Crustacea. J Crustac Biol 40: 122-130.

McMurdie PJ, Holmes S (2013) phyloseq: an R package for reproducible interactive analysis and graphics of microbiome census data. PLOS ONE 8:e61217

Meres NJ, Ajuzie CC, Sikaroodi M, Vemulapalli M, Shields JD, Gillevet PM (2012) Dysbiosis in epizootic shell disease of the American lobster (Homarus americanus). J Shellfish Res 31:463-472

Ooi MC, Goulden EF, Smith GG, Bridle AR (2019) Haemolymph microbiome of the cultured spiny lobster Panulirus ornatus at different temperatures. Sci Rep 9: 1677

*Palleroni NJ (1981) Introduction to the Family Pseudomonadaceae. In: Starr MP, Stolp H, Trüper HG, Balows A, Schlegel HG (eds) The prokaryotes. Springer, Berlin, p 655-665.

* Palleroni NJ (2008) The genus Pseudomonas. In: Goldman E, Green LH (eds) Practical handbook of microbiology. CRC Press, Boca Raton, FL, p 251-262.

Pechal JL, Crippen TL, Tarone AM, Lewis AJ, Tomberlin JK, Benbow ME (2013) Microbial community functional change during vertebrate carrion decomposition. PLOS ONE 8:e79035 
Pérez-Pascual D, Lunazzi A, Magdelenat G, Rouy Z and others (2017) The complete genome sequence of the fish pathogen Tenacibaculum maritimum provides insights into virulence mechanisms. Front Microbiol 8:1542

Pujalte MJ, Lucena T, Ruvira MA, Arahal DR, Macián MC (2014) The Family Rhodobacteraceae. In: Rosenberg E, DeLong EF, Lory S, Stackebrandt E, Thompson F (eds) The prokaryotes. Springer, Berlin, p 439-512.

Quast C, Pruesse E, Yilmaz P, Gerken J and others (2013) The SILVA ribosomal RNA gene database project: improved data processing and web-based tools. Nucleic Acids Res 41:D590-D596

Quinn RA, Metzler A, Smolowitz RM, Tlusty M, Chistoserdov AY (2012) Exposures of Homarus americanus shell to three bacteria isolated from naturally occurring epizootic shell disease lesions. J Shellfish Res 31:485-493

Quinn RA, Smolowitz R, Chistoserdov AY (2013) Cultureindependent analysis of bacterial communities in hemolymph of American lobsters with epizootic shell disease. Dis Aquat Org 103:141-148

Reardon KM, Wilson CJ, Gillevet PM, Sikaroodi M, Shields JD (2018) Increasing prevalence of epizootic shell disease in American lobster from the nearshore Gulf of Maine. Bull Mar Sci 94:903-921

Romano P, Fabritius H, Raabe D (2007) The exoskeleton of the lobster Homarus americanus as an example of a smart anisotropic biological material. Acta Biomater 3: 301-309

Semedo M, Song B (2020) From genes to nitrogen removal: determining the impacts of poultry industry wastewater on tidal creek denitrification. Environ Sci Technol 54: 146-157

Shields JD, Overstreet RM (2007) Diseases, parasites, and other symbionts. In: Kennedy V, Cronin LE (eds) The blue crab Callinectes sapidus. Maryland Sea Grant, College Park, MD, p 299-417

Shields JD, Stephens FJ, Jones B (2006) Pathogens, parasites and other symbionts. In: Bruce P (ed) Lobsters: biology, management, aquaculture and fisheries. WileyBlackwell, Hoboken, NJ, p 146-204

Shields JD, Wheeler KN, Moss J, Somers B, Castro K (2012) The '100 Lobsters' Project: a cooperative demonstration project for health assessments of lobsters from Rhode Island. J Shellfish Res 31:431-438

Sikaroodi M, Gillevet PM (2012) Quality control in multi-tag pyrosequencing of microbial communities. Biotechniques 53:381-383

Sleeter TD, Boyle PJ, Cundell AM, Mitchell R (1978) Rela-

Editorial responsibility: Fuhua Li,

Qingdao, PR China tionships between marine microorganisms and the wood-boring isopod Limnoria tripunctata. Mar Biol 45: 329-336

Stratil SB, Neulinger SC, Knecht H, Friedrichs AK, Wahl M (2013) Temperature-driven shifts in the epibiotic bacterial community composition of the brown macroalga Fucus vesiculosus. Microbiol Open 2:338-349

Sun WW, Zhang XX, Wan WS, Wang SQ and others (2017) Tumor necrosis factor receptor-associated factor 6 (TRAF6) participates in anti-lipopolysaccharide factors (ALFs) gene expression in mud crab. Dev Comp Immunol 67:361-376

Suzuki M, Rappé MS, Giovannoni SJ (1998) Kinetic bias in estimates of coastal picoplankton community structure obtained by measurements of small-subunit rRNA gene PCR amplicon length heterogeneity. Appl Environ Microbiol 64:4522-4529

Tall BD, Fall S, Pereira MR, Ramos-Valle M and others (2003) Characterization of Vibrio fluvialis-like strains implicated in limp lobster disease. Appl Environ Microbiol 69:7435-7446

* Tamas I, Smirnova AV, He Z, Dunfield PF (2014) The (d)evolution of methanotrophy in the Beijerinckiaceae-a comparative genomics analysis. ISME J 8:369-382

* Waller JD, Wahle RA, McVeigh H, Fields DM (2017) Linking rising $p \mathrm{CO}_{2}$ and temperature to the larval development and physiology of the American lobster (Homarus americanus). ICES J Mar Sci 74:1210-1219

Welsh JE, King PA, MacCarthy E (2011) Characterization of a biofilm bacterium from a recirculation system for European lobster (Homarus gammarus). Aquaculture 318: 458-463

Wendling CC, Batista FM, Wegner KM (2014) Persistence, seasonal dynamics and pathogenic potential of Vibrio communities from Pacific oyster hemolymph. PLOS ONE 9:e94256

Wickham H, Chang W (2008) ggplot2: an implementation of the grammar of graphics. R package version 0.7. http:// CRAN.R-project.org/package=ggplot 2

* Williams SL, Jensen RV, Kuhn DD, Stevens AM (2017) Analyzing the metabolic capabilities of a Vibrio parahaemolyticus strain that causes early mortality syndrome in shrimp. Aquaculture 476:44-48

Zhao DH, Sun JJ, Liu L, Zhao HH and others (2009) Characterization of two phenotypes of Photobacterium damselae subsp. damselae isolated from diseased juvenile Trachinotus ovatus reared in cage mariculture. J World Aquacult Soc 40:281-289

Submitted: July 24, 2020; Accepted: November 23, 2020

Proofs received from author(s): February 9, 2021 